CERN LIBRARIES, GENEVA | ||||||||||||||||||||||||||||||||||||||||||||||||||||||||||||||||||||||||||||

CM-P00065590
Corn en $3 i-2$

C. $(9 ; 31)$

CERN-COMPUTING \& NETWORKS DIVISION

$\mathrm{CN} / 91 / 7$

July 1991

VICbus: VMEbus Inter-Crate Bus

A Versatile Cable Bus

Christopher F. PARKMAN

(Presented at the Real-Time 91 Conference, Jülich, 24-28 June 1991) 


\title{
VICbus: VME Inter-Crate Bus A Versatile Cable Bus
}

\author{
Christopher F.Parkman \\ Computing and Networks Division, CERN, 1211 Geneva 23, Switzerland
}

\begin{abstract}
VICbus is a multiplexed, multi-master cable bus primarily intended for interconnecting backplane bus systems, such as VMEbus, but which may also be used wherever a high performance, general purpose cable bus is required. It is implemented using differential line transmission to interconnect up to 31 devices on a total cable length of 100 metres.

Both compelled (asynchronous) and non-compelled (synchronous) data transmission protocols are specified. The compelled protocol is essential when the data transfer protocols of VMEbus backplanes are to be interlocked for software transparent operation, but may also be used for transfers between memories within VICbus devices, not involving associated backplanes. The non-compelled protocol is used in the latter case, when performance is a major factor.

An efficient bus arbitration mechanism takes into consideration the long signal propagation times on cables. A simple interrupt mechanism is specified permitting the transparent use of interrupts on interconnected VMEbus backplanes.
\end{abstract}

Full redundancy of operation avoiding single points of failure may be achieved allowing the construction of "robust", fault-tolerant systems as well as permitting the in-service connection and disconnection of devices with only minor perturbation.

VICbus has been adopted for future international standardisation.

\section{INTRODUCTION}

With notable exceptions such as Fastbus[1], backplane bus systems have traditionally been restricted to implementation in a single crate ${ }^{1}$. In many systems this is a considerable limitation either because it allows insufficient space for the required functionality, or because of a requirement for system redundancy. The inevitable consequence has been the proliferation of proprietary crate extenders and inter-crate connections.

VICbus owes its origins to the recognition by a group of VMEbus[2] users, frustrated by this multiplicity of inter-crate connections, that a standard inter-crate bus would bring many benefits to the users of complex, multi-crate VMEbus systems. Work began in 1986 within this group (the ESONE ${ }^{2}$ VMEbus Working Group) to define basic functional requirements[3], and continues under the aegis of a Joint

1 The term "crate" is used to describe the basic enclosure of a backplane bus system, housing backplane(s), card guides, power supplies and cooling.

2 ESONE: European Standards On Nuclear Electronics committee of European laboratories.
Technical Committee of the ISO/IEC ${ }^{3}$ with the final aim of international standardisation.

Because of its VMEbus orientation, and of the relatively venerable nature of that standard, a pragmatic approach has been taken to speed the standardisation effort, and no fundamental new concepts are proposed: copper cables, available silicon and proven transmission techniques. In addition, advantage has been taken of the generous decision of Creative Electronic Systems SA to allow the low-level protocols of their proprietary inter-crate bus, "VMVbus"[4][5], to be used as a basis for the standardisation. Work has not only been concentrated on refining and enhancing these protocols, but also on adding extra functionality.

The VICbus Specification[12] foresees the use of features currently under study for the enhancement of the VMEbus[6][7].

Whilst the name VICbus is an acronym of "VME InterCrate Bus", the specification makes no basic assumptions that prevent its use for connecting backplane bus systems other than VMEbus, combinations of such systems, or even the design of stand-alone devices. (A "device" in VICbus terminology is either an interface to a backplane bus system or a stand-alone "box"). The specification includes a "normative" annex describing the essential features of a VICbus to VMEbus interface.

\section{DATA TRANSFER PROTOCOLS}

VICbus implements two protocols on the data transfer bus (DTB): compelled and non-compelled. The compelled protocol is fully handshaken permitting both the master and the slave to control the timing of a transfer which, in addition, may be interlocked with that of a backplane bus. The non-compelled protocol (of which VICbus has two variants), allows faster data transfers by eliminating the handshake and hence the cable delay, but cannot be used for interlocked, "transparent" transfers.

The concept of "transparent" operation is essential to VICbus, and permits the extension of a single VMEbus crate system into a multi-crate system with only minor changes to the software. Essentially this implies that a slave module could be moved from one crate to another, with the master being only aware of a change of address (apart from some system initialisation).

The practical implementation of VICbus has necessitated the extensive use of multiplexing. Thus a data transfer always starts with an address phase during which the multiplexed

3 International Organization for Standardization/International Electrotechnical Commission, Joint Technical Committee 1 , Sub-Committee 26 (Microprocessors), Working Group 8. 
Address/Data lines (AD31-AD00) carry a 32 bit address, and the Identification Lines (ID4-ID0) carry a Device Number (DN4-DN0) in order to select one of 31 devices. ( $D N=0$ indicates a broadcast or broadcall). Also, during the address phase, the multiplexed Control Lines (CL3-CLO) carry information identifying the protocol and data byte alignment to be used during the current cycle.

This initial address phase is followed by a data phase during which the ADn lines carry a 32 bit data word, the IDn lines further addressing information, and the CLn lines byte selection, data direction (read or write), and pre-decoded block transfer information.

\section{Compelled protocol}

Figure 1 illustrates the compelled protocol. The timing information is carried between the bus master and the participating slave or slaves on the Address Strobe (AS), Data Strobe (DS), and WAIT lines.

When a master starts a data transfer it will first place the required address phase signals onto the IDn, $A D n$ and $C L n$ lines, to identify the slave (or slaves), the internal address within the slave, and the cycle type (protocol and byte alignment), which it will then qualify by asserting AS. All on-line slaves monitor this information and decide whether they are able and required to participate in the cycle. After a specified time, the master proceeds to the data phase and removes the address phase signals from the IDn, ADn and CLn lines without waiting for any slave response. Thus the address phase is always non-compelled.

The master continues by placing the required data phase signals on the IDn, $A D n$ and CLn lines and asserting DS, to which all participating slaves reply by asserting WAIT.

In a read cycle, the slave will retrieve the required data and place it on the ADn lines. This data may come from a location within the device itself, or may be fetched by means of a cycle generated on an associated backplane bus. At this time, the slave provides status information to the master, if necessary, by asserting the Slave Error (SERR) line to indicate the success, or otherwise, of the transaction. The slave then deasserts WAIT to signal to the master that it may latch the data and the slave response information. Since the specified line driver technology allows wired-OR logic, the WAIT line will remain asserted until deasserted by all participating slaves.

In a write cycle, the master will place the data onto the $\mathrm{ADn}$ lines together with the data phase information, and assert DS. The participating slave will acknowledge assertion of DS by asserting WAIT, proceed to latch the data, and will assert or deassert SERR, as required, and deassert WAIT. On recognition of the deassertion of WAIT, the master knows that all participating slaves have accepted the data, and can deassert DS. Read and write transfers may be intermixed at will within the one cycle. When the data transfers are complete the master terminates the cycle by deasserting AS.

The VICbus timing rules take account of the "wired-OR glitch" problem by making the necessary allowance (i.e. twice the cable propagation delay), in appropriate hold times, to ensure that lines are stable before the next action is taken.



Figure 1. Compelled protocol

\section{Non-compelled protocols}

Unlike the compelled protocol, the non-compelled protocols exchange no timing information between master and slave (with an important exception, see below) thereby avoiding the cable delay inherent in the handshaken protocol. The VICbus specification describes two variants: NonCompelled 1 (NC1) and Non-Compelled 2 (NC2).

The NCl protocol (illustrated in Figure 2) provides the fastest means of data transport between VICbus devices. The master expects no response from any slave, simply assuming its existence and, after the non-compelled address broadcast, toggles the DS line at a pre-determined rate, asserting and deasserting the $A D$ lines as required. The rate at which the slave is able to accept non-compelled transfers (the DS toggling frequency) is signalled to the master in the Device Identification Registers (see below).

Note that because of the lack of slave responses, the $\mathrm{NC1}$ protocol is limited to write transfers only.

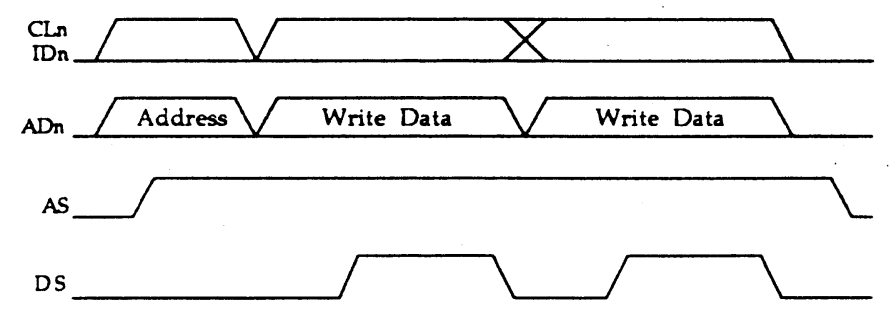

Figure 2. Non-Compelled 1 protocol

The NC2 protocol provides more security than the $\mathrm{NC} 1$ by following the address phase with an initial compelled data transfer to confirm the existence and capability of the slave. The protocol observed in subsequent data transfers is similar to $\mathrm{NCl}$, with the addition of "pipelined" slave responses. In these responses, the WAIT line is asserted and deasserted by the slave in reply to each assertion of DS by the master, but is not used to control the timing of the transfer. The rate at which the slave toggles the WAIT line may, if necessary, be slower than the rate at which the master toggles DS. The round-trip cable delay inherent in the Compelled protocol is thereby eliminated, but the master is able to confirm the success, or otherwise, of the transfers. 
Unlike the NC1 protocol, the NC2 may be used for data transfers in both directions, read and write, but in one direction only in any one cycle.

$\mathrm{NC1}$ transfers with $\mathrm{DN}=0$ indicate that multiple slaves may receive the data simultaneously, but such transfers are not allowed in NC2 because of the pipelined responses. Devices, however, are allowed to "spy" on data transfers, with no interaction in the timing control, whatever the protocol being used.

\section{Cycle types}

Table I summarises the six VICbus cycle types which are distinguished by the value of the CLn lines during the address phase.

Data transfers whose timing is interlocked with that of a backplane bus, are known as transparent cycles and use the compelled protocol. Those which are used to access slaves within devices (i.e. which do not concern any associated backplane bus), are known as direct cycles and may use either the compelled or non-compelled protocols.

Table I

Cycle types

\begin{tabular}{|l|l|l|l|}
\hline Cycle type & Protocol & $\begin{array}{l}\text { Allgn- } \\
\text { ment }\end{array}$ & \multicolumn{1}{|c|}{ A pplication } \\
\hline $\begin{array}{l}\text { Direct } \\
\text { Compelled }\end{array}$ & Compelled & $\begin{array}{l}\text { Byte } \\
\text { lane }\end{array}$ & $\begin{array}{l}\text { Transfers to slaves within } \\
\text { devices with handshake } \\
\text { control. }\end{array}$ \\
\hline $\begin{array}{l}\text { Direct Non- } \\
\text { Compelled 1 }\end{array}$ & $\begin{array}{l}\text { Non- } \\
\text { compelled }\end{array}$ & $\begin{array}{l}\text { Byte } \\
\text { lane }\end{array}$ & $\begin{array}{l}\text { Transfers to slaves within } \\
\text { devices with no handshake } \\
\text { control. }\end{array}$ \\
\hline $\begin{array}{l}\text { Direct Non- } \\
\text { Compelled 2 }\end{array}$ & $\begin{array}{l}\text { Non- } \\
\text { compelled }\end{array}$ & $\begin{array}{l}\text { Byte } \\
\text { lane } \\
\text { Transfers to slaves within } \\
\text { devices with no immediate } \\
\text { handshake control (except } \\
\text { for the first transfer) the } \\
\text { slave's responses being } \\
\text { pipelined. }\end{array}$ \\
\hline $\begin{array}{l}\text { Transparent } \\
\text { VME-A64 }\end{array}$ & Compelled & VMEbus & $\begin{array}{l}\text { A VMEbus A64 cycle is } \\
\text { transmiued over VICbus. }\end{array}$ \\
\hline $\begin{array}{l}\text { Transparent } \\
\text { VME }\end{array}$ & Compelled & VMEbus & $\begin{array}{l}\text { VMEbus A32, A24 or A16 } \\
\text { cycles are transmitted over } \\
\text { VICbus. }\end{array}$ \\
\hline $\begin{array}{l}\text { Transparent } \\
\text { BLA }\end{array}$ & Compelled & $\begin{array}{l}\text { Byte } \\
\text { lane }\end{array}$ & For future applications. \\
\hline
\end{tabular}

Data may be transported across VICbus with either the byte alignment specified by VMEbus (VME), or with the "byte lane" alignment (BLA), as shown in Tables II and III.

Table II

VMEbus byte alignment

\begin{tabular}{|l|c|c|c|c|}
\cline { 2 - 5 } \multicolumn{1}{c|}{} & D31-D24 & D23-D16 & D15-D08 & D07-D 00 \\
\hline byte (0) & - & - & byte(0) & - \\
\hline byte (1) & - & - & - & byte(1) \\
\hline byte (2) & - & - & byte(2) & - \\
\hline byte (3) & - & - & - & byte(3) \\
\hline byte (0-1) & - & - & byte(0) & byte(1) \\
\hline byte (1-2) & - & byte(1) & byte(2) & - \\
\hline byte (2-3) & - & - & byte(2) & byte(3) \\
\hline byte (0-2) & byte(0) & byte(1) & byte(2) & - \\
\hline byte (1-3) & - & byte(1) & byte(2) & byte(3) \\
\hline byte (0-3) & byte(0) & byte(1) & byte(2) & byte(3) \\
\hline byte (4-7) & byte(4) & byte(5) & byte(6) & byte(7) \\
\hline
\end{tabular}

* VMEbus D64 transfer
Table III

Byte Lane Alignment

\begin{tabular}{|c|c|c|c|c|}
\cline { 2 - 5 } \multicolumn{1}{c|}{} & D31-D24 & D23-D16 & D15-D08 & D07-D00 \\
\hline byte (0) & byte(0) & - & - & - \\
\hline byte (1) & - & byte(1) & - & - \\
\hline byte (2) & - & - & byte(2) & - \\
\hline byte (3) & - & - & - & byte(3) \\
\hline byte (0-1) & byte(0) & byte(1) & - & - \\
\hline byte (1-2) & - & byte(1) & byte(2) & - \\
\hline byte (2-3) & - & - & byte(2) & byte(3) \\
\hline byte (0-2) & byte(0) & byte(1) & byte(2) & - \\
\hline byte (1-3) & - & byte(1) & byte(2) & byte(3) \\
\hline byte (0-3) & byte(0) & byte(1) & byte(2) & byte(3) \\
\hline
\end{tabular}

Note that the transfer of VMEbus A64 cycles[6][7] over VICbus, necessitating the use of the first data phase of the transfer for the extra 32 address bits, is indicated by a specific cycle type. VMEbus D64 cycles[6][7] are transmitted using the "natural" order of low order bits (D31-D00) followed by high order bits (D63-D32), but require no special cycle type.

\section{BUS ARBITRATION}

Since it is impractical to use distributed arbitration on a cable bus owing to the relatively long signal propagation times, VICbus has adopted a classical bus request/bus grant mechanism with prioritisation by daisy-chain with, however, a significant enhancement. Every device, when it becomes bus master, also assumes the function of arbiter which thereby moves from device to device, or rotates through the system.

This scheme of a "rotating" arbiter reduces the mean arbitration time, and provides automatic "fairness", (i.e. prevents any one device from taking an undue share of the bus bandwidth). In addition, the multiplicity of arbiters ensures that the inconvenience of a unique arbiter as a potential singlepoint-of-failure can be avoided in fault-tolerant systems.

Unlike VMEbus, only one bus request line is implemented, to limit the total number of lines in a practical cable implementation and to avoid additional prioritisation daisy-chains, with their associated complexity in robust systems.

\section{Arbitration protocol}

The VICbus arbitration protocol requires that the requester asks the arbiter for DTB mastership by asserting the Bus Request (BR) line. It then waits until it receives the assertion of the Bus Grant (BG) line before asserting Bus Busy (BBSY) and deasserting $B R$. On receipt of the assertion of BBSY from the requester, the arbiter deasserts $B G$. Non-requesting devices pass the BG signal on to the next device.

In the case of multiple, simultaneous requests, the priority for bus allocation is decided by the position of the requester on the bus grant daisy-chain relative to the arbiter: those physically nearer having the higher priority.

In the rotating arbitration scheme, the state of an additional line, Arbiter Present (AP), indicates the presence of the "current" arbiter. When the current arbiter grants bus mastership to a requesting device it deasserts $\mathrm{AP}$ and so passes the arbiter function to the new master, which then asserts AP 
to signal its acceptance. The BG line must form a continuous loop in order that devices anywhere on the bus, relative to the current arbiter, can monitor the BG signal. Therefore an additional line, BGLOOP, carries the BG signal from one end of the daisy-chain to the other.

Should the rotation mechanism fail or the current arbiter be removed through reconfiguration, such that AP remains deasserted for longer than a specified time, the device carrying the current IRQSEL Generator (see below), which also monitors AP, will itself assume the arbiter function.

Note that although the rotating arbiter scheme is mandatory, it is permitted to fix the arbiter function onto one specific device (by simply not deasserting AP on change of mastership), when fault-finding or for system integration.

\section{INTERRUPTS}

The VICbus Specification describes a simple interrupt mechanism in which 32 interrupt request signals are multiplexed onto eight physical lines by means of a $125 \mathrm{kHz}$, two-phase clock (IRQSEL). Only one interrupter is allowed per interrupt request signal to avoid an additional daisy-chain for prioritisation and the consequent complexity if "hot" reconfiguration is to be implemented (see below). Interrupt acknowledgement is made by means of a dedicated DTB cycle type: "IACK".

\section{IRQSEL Generator}

The IRQSEL Generator provides the $125 \mathrm{kHz}$, two-phase clock required to multiplex the 32 Multiplex Interrupt Request signals (MIRQ31-MIRQ00) onto the eight Interrupt Request lines (IRQ7-IRQ0). In robust systems, a unique IRQSEL Generator would present a single-point-of-failure but the VICbus Specification calls for the implementation of multiple IRQSEL Generators, and provides an allocation protocol, illustrated in Figure 3.

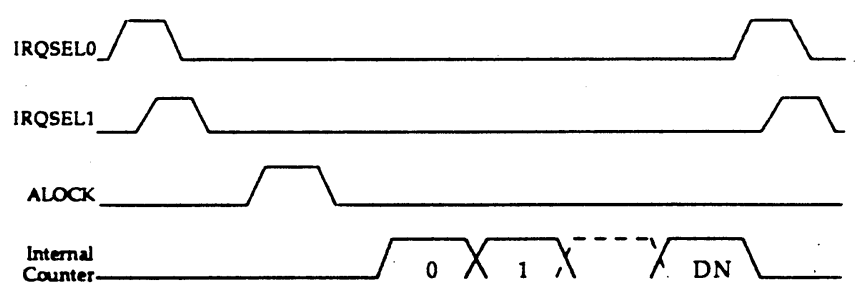

Figure 3. IRQSEL Generator selection

The absence of the IRQSEL Generator is detected by the lack of a transition of either of the IRQSEL clocks for a certain time. All potential IRQSEL Generators will monitor these signals, and if a failure is sensed will assert the Arbitration Lock signal, "ALOCK" (which is also used, as its name implies, to inhibit the VICbus arbitration mechanism during "hot" connection and disconnection - see below).

On detection of the deassertion of ALOCK, all devices equipped with potential IRQSEL Generators count periods of $64 \mu$ s. If such a device counts a number of these periods equal to the value of its own Device Number without seeing the IRQSEL clocks restart, it will itself assume the function of IRQSEL Generator and start to drive them.

\section{Interrupt protocol}

When one device wishes to interrupt another, its interrupter will assert one of the multiplexed interrupt request signals (MIRQn) assigned to it. On receipt of this assertion, the interrupt handler assigned to monitor this signal can take a number of actions such as signalling the event to an embedded processor by means of a flag or local interrupt, or propagating it onto the interrupt mechanism of an associated backplane bus.

The interrupt handler may then proceed to request bus mastership and to fetch a related vector using an IACK cycle (which uses the standard DTB compelled protocol). This cycle will carry an Interrupter Number (IN) equal to the number of the MIRQn in question to identify the interrupter being acknowledged, rather than a Device Number (DN) as in a normal DTB compelled read.

VICbus interrupt vectors may be 8,16 or 32 bits wide, right aligned, similar to VMEbus. The interrupt request may be reset directly by the IACK cycle (i.e. Release-OnACKnowledgement - ROACK in VMEbus terms), or a master associated with the interrupt handler may make a specific action within the requesting device via a standard DTB cycle, which will indirectly reset the request (Release-On-RegisterAccess - RORA).

In addition to the two VMEbus-like interlocked actions described above, a requester may simply assert and deassert its MIRQn in a synchronous, protocol-less manner (subject to a specified minimum duration) requiring no further action by an interrupt handler (i.e. no IACK cycle). This mode (ReleaseOn-Synchronous-Action - ROSA) is intended for process synchronisataion, as well as to minimise interrupt latency during cable connection and disconnection in robust systems (see below).

\section{REGISTERS}

VICbus direct data transfer cycles access slaves within devices, with no action on any associated backplane bus. The slave resources are collectively known as "registers", and consist of a set of mandatory and optional control and status registers, described in the specification, as well as a number of registers which are user definable.

These "registers" are addressed by means of the Register Select signals, RS4-RS0, transmitted on the ID4-ID0 lines during the data phase. The specified control and status registers are single 32-bit locations, but the user definable registers can use the VICbus address signals, A31-A02, together with the byte selection signals, for secondary addressing in order to select locations within blocks of memory.

\section{Broadcall registers}

A number of the control and status registers are intended for broadcall operation in which the device will be operated 
upon, or return its status into, the bit in each register corresponding to its device number. The master, performing a broadcall with the appropriate $\mathrm{RS}$ value, will then retrieve the entire VICbus status in a single 32 bit transfer. These "broadcall registers" (see Table IV), are optional and their functions are replicated in the mandatory Control and Status Register for the convenience of simple implementations.

Table IV

Register summary

\begin{tabular}{|c|l|l|}
\hline RS & \multicolumn{1}{|c|}{ Description } & \multicolumn{1}{|c|}{ Status } \\
\hline 0 & Control and Status Register & Mandatory \\
\hline 1 & Online Register (Broadcall) & Optional \\
\hline 2 & Device Operational Register (Broadcall) & Optional \\
\hline 3 & Reserved & Mandatory \\
\hline 4 & Reset Register & Optional \\
\hline 5 & Transparent Register (Broadcall) & Optional \\
\hline 6 & Reserved & Mandatory \\
\hline 7 & Reserved & Mandatory \\
\hline $8-11$ & Device Identification Registers & Mandatory \\
\hline $12-15$ & Reserved & Mandatory \\
\hline $16-31$ & - & $\begin{array}{l}\text { User } \\
\text { definable }\end{array}$ \\
\hline
\end{tabular}

\section{Device Identification Registers}

Four mandatory Device Identification Registers are specified, containing configuration and identification information, and are detailed in Tables V, VI and VII. The manufacturer identification code included in DIR0 will be issued to manufacturers by a central body, whereas the device identification in DIR1 will be designated by the manufacturer himself.

Table V

Device Identification Registers

\begin{tabular}{|c|c|c|c|c|c|}
\hline & \multicolumn{4}{|c|}{ Function } \\
\hline$\overline{R S}$ & $\begin{array}{l}\text { Reg. } \\
\text { ister }\end{array}$ & $\begin{array}{c}\text { Bit } \\
31.24\end{array}$ & $\begin{array}{c}\text { Bit } \\
23.16\end{array}$ & $\begin{array}{c}\text { Bit } \\
15.08\end{array}$ & $\begin{array}{c}\text { Bit } \\
07.00\end{array}$ \\
\hline 8 & DIR0 & \multicolumn{2}{|c|}{ User definable } & Reserved & $\begin{array}{l}\text { Manufacturer } \\
\text { code }\end{array}$ \\
\hline 9 & $\overline{\text { DIR1 }}$ & \multicolumn{2}{|c|}{ User definable } & Reserved & Device type \\
\hline 10 & DIR2 & \multicolumn{2}{|c|}{ User definable } & Reserved & Capability \\
\hline 11 & DIR3 & \multicolumn{2}{|c|}{ User definable } & Reserved & $\begin{array}{c}\text { Non- } \\
\text { compelled } \\
\text { slave speed }\end{array}$ \\
\hline
\end{tabular}

Table VI

DIR 2 functions

\begin{tabular}{|l|l|}
\hline Bit & Capability (bit asserted indicates the capability) \\
\hline 00 & Slave: broadcast and broadcall transfers implemented \\
\hline 01 & Slave: non-compelled 1 transfers implemented \\
\hline 02 & Slave: non-compelled 2 transfers implemented \\
\hline 03 & VMEbus master: A64 transfers implemented \\
\hline 04 & VMEbus master: D64 transfers implemented \\
\hline 05 & Slave: block transfers implemented \\
\hline 06 & The device is a VMEbus interface \\
\hline 07 & Reserved \\
\hline
\end{tabular}

The device capability information in DIR2 is included to allow the system's initialisation procedures to determine the type and capability of a slave device. The slave speed information in DIR3 may be used by a master to optimise transfer rates in non-compelled mode.

Table VII

DIR 3 functions

\begin{tabular}{|c|c|l|}
\hline Bit & Name & \multicolumn{1}{c|}{ Function } \\
\hline $06-00$ & NCSPEED & $\begin{array}{l}\text { This field gives the minimum time (in tens of } \\
\text { ns) between successive assertions of DS which } \\
\text { the device is capable of accepting as a slave in } \\
\text { a non-compelled transfer. }\end{array}$ \\
\hline 07 & $\begin{array}{l}\text { FAST/ } \\
\text { SLOW }\end{array}$ & $\begin{array}{l}\text { When set to zero, the device is capable of } \\
\text { performing non-compelled transactions at the } \\
\text { rate given by bits 06-00. } \\
\text { When set to one, the device is capable of } \\
\text { performing non-compelled transactions at the } \\
\text { fastest rate permitued by the specification. }\end{array}$ \\
\hline
\end{tabular}

\section{IMPLEMENTATION DETAIIS}

\section{Cables and connectors}

The VICbus consists of a cable (or number of cables) totalling 72 twisted pairs connected in a point-to-point fashion from device to device, or from device to terminator. The total cable length is limited to a maximum of 100 metres, for reasons of signal integrity in difficult electrical environments with practical drivers and receivers, and in order to determine certain timing parameters.

Six high density (" $2 \mathrm{~mm}$ ") connectors[13] are used, three for each "direction", mounted on the transition module in a robust system (see below), and on the device itself if faulttolerance is not required.

The specified drivers and receivers normally require the use of a cable having a characteristic impedance of approximately $110 \Omega$ to ensure adequate noise immunity, however, measurements made with a readily available cable[14], compatible with the chosen connectors, which has a considerably lower impedance $(\approx 85 \Omega)$, has shown satisfactory results[9] over the specified cable length.

\section{Line drivers and receivers}

Open-collector, open-emitter, differential line drivers are used to allow the wired-OR logic necessary for broadcast and broadcall operation. Although a maximum of 31 devices may be connected to the cable, currently available transceiver silicon[8] can only support 24. Efforts are being taken to identify the enhancements necessary to implement the full number.

\section{Reset and system failure}

The VICbus carries a global reset line, VICRESET, and a system failure line, DEVFAIL, equivalent in function to the VMEbus SYSRESET* and SYSFAIL* lines, respectively. In addition to the global reset, devices may be selectively reset by asserting the appropriate bit in their Command and Status or Reset Registers. It is worth noting that since selective resets are generated by means of normal DTB cycles, they may be broadcast. 


\section{Online and offline states}

A device may be set into one of three states: onlineenabled, online-disabled and offline. The online-enabled state implies the presence of a associated backplane bus, whereas the online-disabled state is applicable to all devices. The offline state means that a device has been logically removed from the VICbus cable, and consequently will neither respond to, nor will cause any activity on the VICbus.

Table VIII summarises the actions permitted in each of the three states.

Table VIII

Permitted device actions

\begin{tabular}{|c|c|c|c|}
\hline & \multirow{2}{*}{\multicolumn{3}{|c|}{ 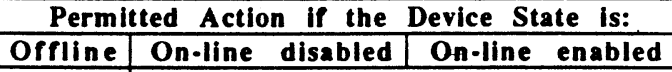 }} \\
\hline Function & & & \\
\hline DTB Master & None & \multicolumn{2}{|c|}{$\begin{array}{c}\text { May generate direct cycles } \\
\text { May generate transparent cycles } \\
\text { May forward cycles from an associated } \\
\text { backplane bus }\end{array}$} \\
\hline DTB Slave & None & $\begin{array}{l}\text { May respond } \\
\text { May not respond to } \\
\text { transparent cycles }\end{array}$ & $\begin{array}{l}\text { o direct cycles } \\
\text { May respond to } \\
\text { transparent cycles } \\
\text { by generating } \\
\text { corresponding } \\
\text { cycles on an } \\
\text { associated backplane } \\
\text { bus }\end{array}$ \\
\hline Requester & None & \multicolumn{2}{|c|}{ May generate bus requests } \\
\hline Interrupter & None & $\begin{array}{l}\text { May generate in } \\
\text { May not forward } \\
\text { interrupts generated } \\
\text { by a device } \\
\text { connected to an } \\
\text { associated backplane } \\
\text { bus, as it cannot } \\
\text { handle IACK cycles }\end{array}$ & $\begin{array}{l}\text { terrupt requests } \\
\text { May forward } \\
\text { interrupts generated } \\
\text { by a device } \\
\text { connected to an } \\
\text { associated backplane } \\
\text { bus }\end{array}$ \\
\hline $\begin{array}{l}\text { Interrupt } \\
\text { Handler }\end{array}$ & None & \multicolumn{2}{|c|}{ May handle interrupts } \\
\hline
\end{tabular}

\section{Timing considerations}

Since the cable propagation velocity $(\approx 5-6 \mathrm{~ns} /$ metre) is an important parameter determining the performance of a cable bus[9], in many cases VICbus specifies two timing parameters: one for cables up to 30 metres in length ("short"), and the other for cables from 30 to 100 metres ("long"). A bit in the Command and Status Register (see above), which is set at system initialisation time, indicates whether the cable is long or short, and the designer may use this information to optimise the timing of the bus protocols. If this refinement is not required, the cable can be simply assumed to be "long".

\section{Fault tolerance and "hot" reconfiguration}

VICbus systems may be designed which are tolerant of failure or deliberate reconfiguration. Such "robust" systems avoid single points of failure and include mechanisms which allow the addition or removal of devices during VICbus operation without major perturbation ("hot" reconfiguration).

The rotating arbitration mechanism avoids the traditional single system arbiter, at the (small) cost of some additional complexity in every master. The IRQSEL clocks, used for multiplexing the interrupt requests, can be replicated in every master (see above), again at the cost of additional logic, not only for the clocks themselves, but also for the necessary allocation mechanism.
The continuity of the cable itself may be guaranteed by the use of a separate transition module, carrying the VICbus cable . connectors, on which all the lines, except BG, are throughconnected. This module remains with the cables even when disconnected from the VICbus device (see Figure 4). In addition, the glitch-free connection and disconnection of bused signals is guaranteed by the specified characteristics of the bus drivers and receivers.

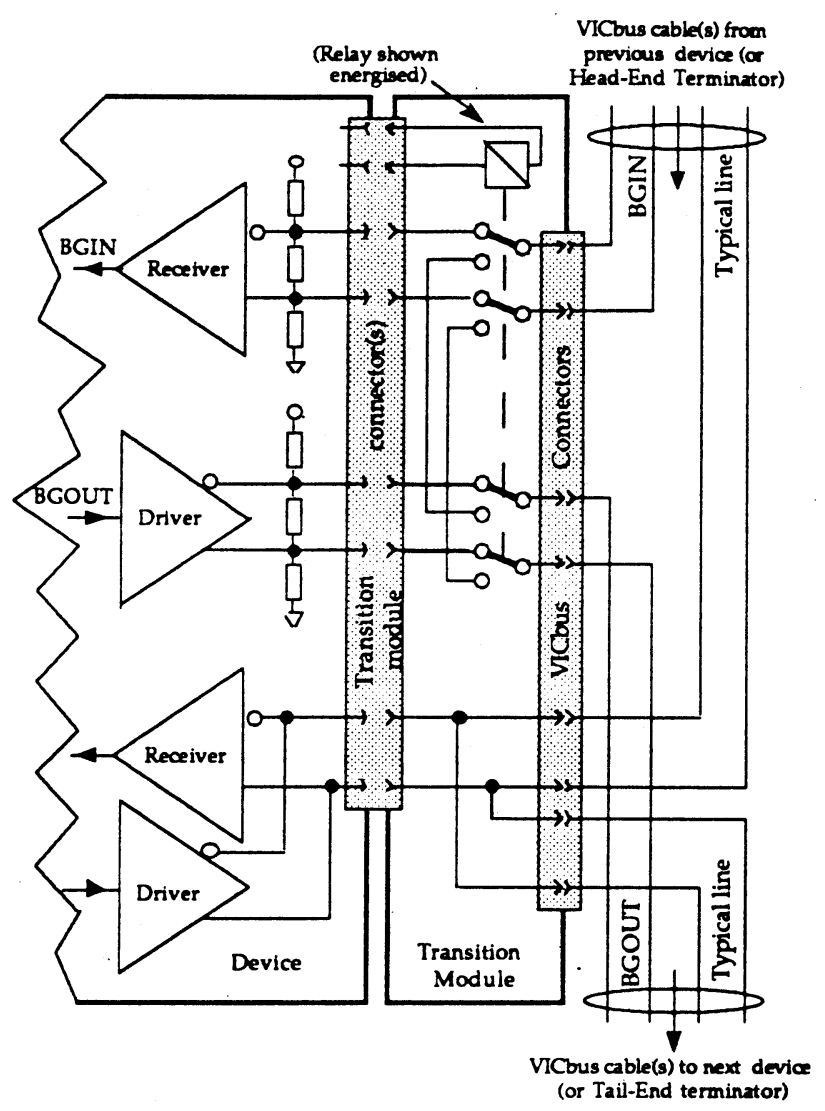

Figure 4. Continuity of lines in robust systems

However, it is the bus grant daisy-chain itself which poses that major problem: how to achieve continuity when a device is powered down, or even removed from the bus? Several possibilities were explored at length during the evolution of the VICbus Specification, but finally the simplest solution was adopted and the necessary ohmic connection is provided by the contacts of an electro-mechanical relay, mounted on the transition module.

The problem of bouncing contacts was overcome by the addition of the Arbitration Lock (ALOCK) line which, when asserted, inhibits the arbitration process until the relay contact chatter has subsided. However, the latency thereby introduced into bus arbitration is undesirable in a real-time system (although it is to be hoped that such reconfiguration does not happen too often), but the judicious use of ROSA interrupters can ensure that at least interrupts can still be passed during the several milliseconds when the BG continuity relay contacts are settling. 
The electrical terminators are clearly also potential singlepoints-of-failure and in robust systems must be powered in an uninterruptable manner, independently of any device. The possibility of distributing terminator power via the cable was explored, but rejected not only because of the impracticality of passing the required current along signal cables, but of the means to ensure a perturbation-free supply in case of "hot" connection or disconnection.

\section{Physical layout}

The VICbus Specification makes no attempt to impose any other part of the physical implementation than the cable and its connectors, since devices may be either interfaces to backplane bus systems or stand-alone "boxes". In the former case their physical form is dependent on the bus in question, whereas in the latter case, they may be constructed in any suitable format.

Robust systems will require the implementation of a transition module, as described above, to carry the cable " $T$ " connections and the BG continuity relays, and which is in turn connected to the device in an implementation-dependent manner. It is this module, and its implications on cable stub ${ }^{1}$ length that will place restrictions on the physical implementation in such cases.

A passive transition module must be mounted very close to the drivers and receivers to respect the specified stub length of $100 \mathrm{~mm}$. In a typical VMEbus system this implies that it will be attached to the front-panel (unless the user definable pins on connector $\mathrm{P} 2$ are available, to which it could be connected by means of a very short cable). However, it is conceivable to mount the drivers and receivers remotely from the body of the VICbus interface logic, in such a manner that the transition module can be more easily connected.

\section{ASPECTS OF INTERFACING TO BUS SYSTEMS}

The VICbus Specification contains a normative annex (i.e. one which is part of the formal specification) giving the regulations and guide-lines essential to the interconnection of VMEbus crates by means of VICbus, additional to the regulations contained in the body of the document. No attempt is made to detail a particular implementation. It is intended to add similar annexes describing interfaces to other backplane bus systems, should they become necessary at a later date.

\section{Mapping}

The annex permits the mapping of VMEbus address and address modifier (AM) information, i.e. the address and the AM code may be different in the destination crate to that in the source crate. However, the mapping of the AM codes should be done with caution, since they can also carry data width and protocol information.

\section{Interrupts}

The VICbus interrupt mechanism allows the use of interrupts in a largely transparent manner, between an

1 The length of the connection from the cable per se to the driver/receiver combination (the "leg of the T"). interrupter on one VMEbus backplane and an interrupt handler on another, connected via VICbus. It should be noted, however, that the "hardware" interrupt mechanism is included for the sake of simple slave devices, and that the use of the DTB for message passing should be envisaged as a more general and efficient means of transporting exceptions from crate to crate.

The VMEbus interrupt mechanism transports two items of information from interrupter to interrupt handler: the interrupt level (IRQ1* to IRQ7*) and a status/id (or vector). The former is transported across VICbus by the mapping of VMEbus interrupt request line to VICbus MIRQn signal, known to the system at configuration time, and the vector by the use of the IACK DTB cycle.

\section{Arbitration dead-lock}

When interconnecting backplane bus systems the effect of adding a second level of arbitration must be carefully considered, or contention for access to the inter-device paths will result in undue loss of bandwidth. The efficient resolution of potential arbitration "dead-locks" when using interlocked cycles (transparent transfers) will be a major factor in determining the overall system performance.

Assume that a VMEbus module has requested mastership of its local VMEbus, which has been granted by the VMEbus arbiter, and the module has asserted BBSY*. If it now attempts a data cycle across VICbus, it will in turn require VICbus mastership. If at the same time the VICbus interface in the same crate requires mastership of the VMEbus in order to pass an incoming VICbus cycle, the system will lock-up and neither of the two contending cycles will be executed.

With the present VMEbus specification[2] the only means available to the VICbus interface to resolve this situation is to abort the local bus cycle by returning BERR*. The VMEbus master will then relinquish bus mastership so allowing the VICbus interface to become master and to complete the pending VICbus cycle.

However, the "error" signalled by BERR* is not fatal, and a retry may be made with a large chance of success. Thus the VMEbus master must be able to distinguish between an assertion of BERR* due to events local to its VMEbus such as access to non-existent memory, etc., and one due to the inter-crate bus arbitration dead lock resolution, and treat them accordingly.

The RETRY* line, introduced into the proposed revision of the VMEbus specification[6], enables a VICbus interface to signal the dead lock resolution situation directly to the VMEbus master.

\section{PERFORMANCE}

\section{Compelled protocol}

The minimum overhead introduced by VICbus into every cycle of a block transfer between crates using the Compelled Protocol, may be calculated from the specified minimum timing parameters and the cable length for both short ( $0<$ length $<30 \mathrm{~m})$ and long $(30 \leq$ length $<100 \mathrm{~m})$ cables. Note 
that the conservative value of $6 \mathrm{~ns} / \mathrm{m}$ for the propagation velocity of a twisted pair cable is used in the VICbus Specification.

Table IX presents these figures for a number of cable lengths, both in terms of an overhead per cycle, and as a data transfer rate.

Table IX

Compelled protocol direct block transfers: calculated performance (DS to DS)

\begin{tabular}{|c|c|c|}
\hline Cable Length (m) & Overhead (ns) & Rate $^{*}$ (Mbyte/s) \\
\hline 3 & 136 & 29.4 \\
\hline 10 & 220 & 18.2 \\
\hline 20 & 340 & 11.8 \\
\hline 30 & 460 & 8.7 \\
\hline 50 & 700 & 5.7 \\
\hline 100 & 1300 & 3.1 \\
\hline
\end{tabular}

* Zero access time memory is assumed

The results of measurements[10] made using a real implementation of a VICbus/VMEbus interface[11] to connect two VMEbus crates are given in Table X. (Note that unlike Table $X$, where zero access time memory is assumed, the measurements in Table $X$ were made with a memory of $150 \mathrm{~ns}$ access time in transparent cycles, and $100 \mathrm{~ns}$ in direct cycles).

Table X.

Compelled protocol block transfers: measured performance (DS to DS)

\begin{tabular}{l|c|c|}
\hline Cycle type & Block transfer & Rate (Mbyte/s) \\
\hline \multirow{3}{*}{ Transparent } & Read $^{* *}$ & 6.1 \\
\cline { 2 - 3 } & Write $^{* * *}$ & 6.1 \\
\cline { 2 - 3 } & Read & 4.4 \\
\cline { 2 - 3 } & Write $^{* * *}$ & 4.9 \\
\hline \multirow{2}{*}{ Direct } & Read $^{* *}$ & 8.0 \\
\cline { 2 - 3 } & Write $^{* *}$ & 8.9 \\
\hline \multirow{2}{*}{ ** Target VMEbus memory DS* to DTACK access time: 150 ns } \\
Target memory DS* to DTACK* access time: 100 ns \\
With a prefetch mechanism on read and write posting on write
\end{tabular}

\section{Non-compelled protocol}

In a similar manner to the above, the theoretical maximum data transfer rates between VICbus devices using the noncompelled protocols may be calculated, again based on the minimum specified timing parameters. Table XI gives these figures (zero access time memory assumed), and Table XII practical measurements (memory access time $100 \mathrm{~ns}$ ). In this case, since the protocols are not handshaken, the rates are less dependent on cable length. The difference between the rates obtained with short and long cables being due solely to the different account taken in the specification of the effects of signal distortion in the two cases.

Table XI

Non-Compelled 1 protocol: calculated data transfer rates (DS to DS)

\begin{tabular}{|l|c|}
\cline { 2 - 2 } \multicolumn{1}{c|}{} & Rate $^{*}$ (Mbyte/s) \\
\hline Short cable $(0<$ length $<30 \mathrm{~m})$ & 33 \\
\hline Long cable $(30<$ length $<100 \mathrm{~m})$ & 19 \\
\hline
\end{tabular}

* Zero access time memory is assumed
Table XII

Non-Compelled 1 protocol: measured data transfer rates (DS to DS)

\begin{tabular}{|l|c|}
\cline { 2 - 2 } \multicolumn{1}{c|}{} & Rate $^{*}$ (Mbyte/s) \\
\hline Short cable $(0$-length<30m) & 14.0 \\
\hline Short cable $(0$ - length $<30 \mathrm{~m})$ & 8.7 \\
\hline
\end{tabular}

* Target memory DS* - DTACK* access time: $100 \mathrm{~ns}$

** With a prefetch mechanism on read and write posting on write

\section{STATUS}

At the time of writing (late-June 1991) the VICbus Specification has been under development by the ISO/IEC Working Group for more than one and a half years, and has undergone a number of major revisions. It is now under consideration by the ISO/IEC JTC1 Sub-Committee 26 at the "Committee Draft" stage, from which it will move, after formal balloting procedures to its adoption as a fully homologated, international standard.

\section{ACKNOWLEDGEMENTS}

The author wishes to emphasise that this paper is a report on the work undertaken by the VICbus Working Group (ISO/IEC JTC1 SC26 WG8):

J.Alexander (S.E.R.C., Daresbury Lab., Warrington, UK)

J.Bovier (C.E.S., Geneva, Switzerland) •

M.Chorowicz (C.E.S., Geneva, Switzerland)

G.Duchi (Bailey Esacontrol, Genoa, Italy)

C.Eck (CERN, Geneva, Switzerland - Convenor)

K.Fründt (Motorola, Munich, Germany)

C.Parkman (CERN, Geneva, Switzerland - Editor)

S.Pri-Tal (Motorola, Tempe, Arizona, USA)

The author would like to express his thanks to his colleagues Christoph Eck and Jørgen Petersen, and to his wife, Vivienne, for their time and patience in reading and criticising this paper.

\section{REFERENCES}

[1] IEEE960-1986, "Fastbus Specification".

[2] IEEE1014-1987, "VMEbus Specification".

[3] C.F.Parkman, "VICbus - "The VME Inter-Crate Bus", in Buscon UK Proceedings, London, October 1987.

[4] Creative Electronic Systems, "Vertical Bus Receiver".

[5] Creative Electronic Systems, "Vertical Bus Emitter".

[6] IEEE P1014-R, revised VMEbus Specification - in preparation.

[7] S.Pri-Tal, "The Future of VMEbus" - in New Backplane Bus Architectures, proceedings, ESONE VMEbus Working Group, CERN, Geneva, March 1990.

[8] Advanced Micro Devices, Am26LS238 Data Sheet.

[9] G.Duchi, Private Communication, March 1991.

[10] M.Chorowicz, Private Communication, June 1991.

[11] Creative Electronic Systems SA, "VIC8251 User's Manual", 1991.

[12] ISO/IEC JTC1 SC26 WG8 (C.F.Parkman - Editor), "The VICbus Specification, Draft Version 1.1", May 1991.

[13] Du Pont Electronics, "Metral Connectors".

[14] Eurel Intemational B.V, "Metral cable specification". 